\title{
Pretreatment with an adenosine $A 1$ receptor agonist and lidocaine: A possible alternative to myocardial ischemic preconditioning
}

\author{
Sarah J. Canyon, PhD, and Geoffrey P. Dobson, PhD
}

From the Department of Physiology and Pharmacology, School of Biomedical Sciences, James Cook University, Queensland, Australia.

Received for publication Aug 2, 2004; revisions received Dec 15, 2004; accepted for publication Dec 23, 2004.

Address for reprints: Geoffrey P. Dobson, $\mathrm{PhD}$, Department of Physiology and Pharmacology, Molecular Science Building, James Cook University, Townsville, Queensland 4811, Australia (E-mail: geoffrey.dobson@ jcu.edu.au).

J Thorac Cardiovasc Surg 2005;130:371-7

$0022-5223 / \$ 30.00$

Copyright (๑) 2005 by The American Association for Thoracic Surgery

doi:10.1016/j.jtcvs.2004.12.052
Objective: The heart possesses an extraordinary ability to remember short episodes of sublethal ischemia and reperfusion (angina), which protects the myocardium and coronary vasculature from a subsequent lethal insult, a phenomenon known as ischemic preconditioning. A therapeutic goal for more than 2 decades has been to develop a pharmacologic mimetic comparable with ischemic preconditioning. Our aim was to investigate the preconditioning effect of a new combinatorial therapy targeting adenosine A1 receptors and voltage-dependent sodium fast channels in the in vivo rat model of regional ischemia.

Methods: Ischemia-reperfusion was achieved by placing a reversible tie around the left coronary artery in anesthetized and ventilated Sprague-Dawley rats $(\mathrm{n}=37)$. Rats were randomly assigned to 1 of 5 groups: (1) saline control $(\mathrm{n}=13)$; (2) ischemic preconditioning $(\mathrm{n}=6)$; (3) lidocaine only $\left(608 \mu \mathrm{g} \cdot \mathrm{kg}^{-1} \cdot \min ^{-1}, \mathrm{n}=\right.$ 5); (4) adenosine A1 receptor agonist 2-chloro-N6-cyclopentyladenosine (CCPA; 5 $\mu \mathrm{g} / \mathrm{kg}, \mathrm{n}=7)$; and (5) CCPA plus lidocaine $(\mathrm{n}=6)$. Ischemic preconditioning was achieved by using 3 cycles of ischemia and reperfusion lasting 3 minutes each. Lidocaine was infused continuously 5 minutes before and throughout 30 minutes of ischemia and ceased at reperfusion. A bolus of CCPA was infused 5 minutes before ligation along with a constant infusion of lidocaine (as above). All animals were reperfused for 120 minutes for infarct size measurement.

Results: Fifty-four percent of saline control rats, $17 \%$ of ischemic preconditioningtreated rats, and $29 \%$ of CCPA-treated rats died during ischemia from ventricular fibrillation. Infarct size of saline control animals was $61 \% \pm 5 \%$. Pretreating with CCPA and lidocaine infusion resulted in no deaths, no severe arrhythmias, and significant infarct size reduction compared with that seen in saline control animals $(P<.05)$. Remarkably, infarct size reduction in CCPA plus lidocaine-treated rats $(12 \% \pm 4 \%)$ was equivalent to that achieved with ischemic preconditioning $(11 \% \pm$ $3 \%$ ), whereas infarct size in rats undergoing CCPA-only and lidocaine-only treatments was $42 \% \pm 7 \%$ and $60 \% \pm 6 \%$, respectively. Although CCPA plus lidocaine treatment reduced heart rate, mean arterial pressure, and systolic pressure during ischemia, no correlation was found between these variables and infarct size reduction.

Conclusion: We conclude that activating adenosine A1 receptor subtype with CCPA and concomitantly modulating sodium fast channels with lidocaine was comparable with ischemic preconditioning and might offer a new therapeutic window to minimize myocardial damage during surgical ischemia and reperfusion.

I schemic preconditioning (IPC) was first described in 1986 by Murry and colleagues, ${ }^{1}$ who reported an infarct size reduction from $29 \%$ to $7 \%$ in anesthetized open-chest dogs after 3 brief episodes of ischemia and reperfusion and 40 minutes of coronary artery occlusion. Two different time frames of precondi- 
tioning have been identified: an early classical window that lasts 1 to 3 hours after the stimulus and a delayed window that develops over many hours and can last up to 12 to 72 hours. ${ }^{2-5}$ In addition, more recently, Zhao and associates ${ }^{6}$ have reported similar infarct-reducing effects by postconditioning the heart a few seconds after reperfusion has commenced.

Given the reluctance of most surgeons and clinicians to precondition an organ in the clinical setting, the ultimate therapeutic goal has been to develop a pharmacologic mimetic of IPC. ${ }^{7}$ Potential triggers include adenosine, opioids, acetylcholine, bradykinin, catecholamines, free radicals, and nitric oxide. ${ }^{4,8-12}$ Adenosine, an endogenous nucleoside, is of particular interest because in addition to triggering early and delayed IPC, ${ }^{5}$ it protects against cellular injury at multiple sites and levels during ischemia and reperfusion. ${ }^{13}$ Adenosine elicits negative chronotropic and dromotropic effects, ${ }^{14,15}$ induces coronary vasodilation, and attenuates the inflammatory response and possibly anticlotting processes. ${ }^{16-19}$ Four known adenosine receptor subtypes (A1, A2a, A2b, and A3) mediate the pleiotropic effects of adenosine, and these receptors are coupled to a wide range of second messenger cascades. ${ }^{16}$ In the heart the adenosine A1 receptor subtype is known to confer protection through inhibitory $G$ protein-coupled pathways, which has been linked to the opening of sarcolemmal adenosine triphosphate (ATP)-sensitive potassium channels, increased potassium conductance, action potential shortening, and reduced $\mathrm{Ca}^{2+}$ entry into ischemic cells. ${ }^{20}$ More recently, the adenosine A1 receptor trigger has been linked to new targets, including the mitochondria ${ }^{21}$ and sarcoplasmic reticulum. ${ }^{22}$ Unlike adenosine A1 (and A3) receptors, cardiac $\mathrm{Na}^{+}$channels have received surprisingly little attention in the preconditioning or pretreatment phenomenon, despite voltagegated $\mathrm{Na}^{+}$channels being important determinants of the cell membrane potential, which is naturally modified toward a more depolarized state during early ischemia. ${ }^{23,24}$

The aim of this study is to examine the effect of coadministering the A1 agonist 2-chloro-N6-cyclopentyladenosine (CCPA) and the voltage-dependent sodium channel modulator lidocaine during acute regional ischemia in the in vivo rat model. Our previous work has shown that a constant intravenous infusion of an adenosine-lidocaine solution administered 5 minutes before and 30 minutes during ischemia protects against death and severe ventricular arrhythmias and reduces infarct size by $40 \%$ in the rat in vivo. ${ }^{25}$ Because $\mathrm{A} 1$ receptor activation has been shown to be highly cardioprotective, ${ }^{16,26}$ we hypothesized that substituting the A1 receptor agonist CCPA for adenosine might demonstrate greater efficacy than administering the adenosine and lidocaine (AL) solution. We report that coadministering CCPA plus lidocaine before and during ischemia completely avoided death, abolished severe arrhythmias, and profoundly reduced infarct size akin to that seen with IPC. The pharmacologic activation of the adenosine A1 receptor subtype and modulation of $\mathrm{Na}^{+}$fast channels might offer an alternative to IPC for reduction of myocardial damage during ischemia and reperfusion.

\section{Materials and Methods \\ Animals and Reagents}

Male Sprague-Dawley rats (330-400 g, fed) were anesthetized with sodium pentabarbitone $(60 \mathrm{mg} / \mathrm{kg}$ administered intraperitoneally), which was administered as required throughout the experiment (ethics approval no. A557). The investigation conforms with the "Guide for Care and Use of Laboratory Animals" published by the US National Institutes of Health (publication no. 85-23, revised 1996). Adenosine, copper II pthalocyanine-tetrasulfonic acid tetrasodium salt (blue dye), triphenyltetrazolium chloride (TTC), and CCPA were obtained from Sigma Aldrich. CCPA has a 10,000fold sensitivity for adenosine A1 than A2 receptor and a subnanomolar receptor affinity. ${ }^{5} \mathrm{CCPA}$ also has a moderately potent antagonistic effect $\left(\mathrm{K}_{\mathrm{i}}=38 \mathrm{nmol} / \mathrm{L}\right)$ on human adenosine A3 receptor. ${ }^{27}$ Lidocaine hydrochloride was purchased as a $2 \%$ solution (ilium).

\section{Surgical Protocol}

The surgical procedure has been described by Canyon and Dobson. ${ }^{25}$ In brief, a tracheotomy was performed, and rats were ventilated at 75 to 80 strokes per minute by with a Harvard Small Animal Ventilator. Blood $\mathrm{PO}_{2}, \mathrm{PCO}_{2}$, and $\mathrm{pH}$ were assessed (Ciba-Corning 865 blood gas analyzer). Body temperature was maintained at $37^{\circ} \mathrm{C}$ by using a homeothermic blanket control unit. The right and left femoral veins and arteries were cannulated for drug infusions, blood collection, and blood pressure monitoring (UFI $1050 \mathrm{BP}$ ) by using a MacLab system (ADInstruments Pty Ltd). A left thoracotomy was performed through the fourth and fifth intercostal spaces. The heart was gently exteriorized to allow for a 6-0 suture to be threaded under the left coronary artery to a reversible snare occluder. Lead II electrocardiographic leads were implanted subcutaneously. Ischemia was confirmed by means of regional cyanosis and swelling downstream of the occlusion, and reperfusion was confirmed by lack of cyanosis and reduced swelling in that region. Any animal with dysrhythmias or a sustained decrease in mean arterial blood pressure of less than $80 \mathrm{~mm} \mathrm{Hg}$ before ischemia was not included. ${ }^{25}$

\section{Experimental Design}

Rats $(\mathrm{n}=37)$ were randomly assigned to one of 5 groups: (1) saline $(0.9 \%)$ control $(\mathrm{n}=13)$; (2) IPC $(\mathrm{n}=6)$; (3) lidocaine only $\left(608 \mu \mathrm{g} \cdot \mathrm{kg}^{-1} \cdot \min ^{-1}, \mathrm{n}=5\right)$; (4) CCPA $(5 \mu \mathrm{g} / \mathrm{kg}, \mathrm{n}=7)$; and (5) CCPA plus lidocaine $(n=6)$. IPC was achieved before coronary artery occlusion with 3 cycles of ischemia and reperfusion, with each transition lasting 3 minutes. Lidocaine was infused continuously 5 minutes before and throughout 30 minutes of regional ischemia and ceased at reperfusion. A bolus of CCPA was infused 5 minutes before ligation alongside a continuous infusion of lidocaine, which was continued during 30 minutes ischemia. All animals were reperfused for 120 minutes for infarct size measurement. The primary end points were death, episodes and duration of 
ventricular arrhythmias, and infarct size. Infarct size reduction is considered one of the gold standard measures of IPC. ${ }^{3}$ Hemodynamics were collected throughout the study and constituted the secondary end points: heart rate, systolic pressure, mean arterial pressure (MAP; Systolic pressure - Diastolic pressure/3 + Diastolic pressure), and rate-pressure product (RPP; Heart rate $\times$ Peak systolic pressure).

\section{Arrhythmia Analysis}

Arrhythmias were analyzed during ischemia and the first 30 minutes of reperfusion. By using the lead II electrocardiographic tracing, the episodes and duration of episodes of ventricular fibrillation (VF) and ventricular tachycardia (VT) were recorded. VF was defined as a signal in which individual QRS deflections could not easily be distinguished from each other and $n$ which rate could no longer be measured, and VT was defined as 4 or more consecutive ventricular premature beats. ${ }^{28}$

Analysis of the Ischemic Area at Risk and Infarct Size After 120 minutes of reperfusion, the coronary artery was reoccluded, and the heart was excised. Blue dye $(3 \mathrm{~mL})$ was flushed retrograde through the aorta and allowed to circulate through the coronary vasculature to delineate the ischemic risk zone. The heart was sliced transversely into 6 or 7 slices of uniform thickness (2 $\mathrm{mm}){ }^{25}$ The slices were weighed and digitally photographed for area measurements. The area left unstained by the blue dye was defined as the left ventricular area at risk (AAR/LV), and the blue-stained region was the perfused area. The slices were then incubated in a $1 \%$ solution of TTC at $37^{\circ} \mathrm{C}$ for 15 minutes, ${ }^{29}$ immersed in formalin, and photographed again. The area of necrosis in the left ventricle (AN/LV) was the region of the slice unstained by TTC (white), and the noninfarcted region was the area of the slice stained by TTC (brick red). Infarct size was defined as the ratio of the area of necrosis to the area at risk (AN/AAR) and expressed as a percentage. ${ }^{25}$

\section{Statistical Analysis}

All values were expressed as means \pm SE of the mean. Infarct size and hemodynamic data were evaluated by using 1-way analysis of variance with a Tukey honestly significant difference post-hoc test. A Mann-Whitney $U$ test was used for comparison of arrhythmia frequency and duration because the variables of VT and VF are not normally distributed. The relationship between RPP and infarct size was examined with the Pearson correlation coefficient.

\section{Results}

Death during ischemia from ventricular arrhythmias occurred in $54 \%$ of saline control animals (7/13 died), $17 \%$ of IPC-treated rats (1/6 died), and 29\% of CCPA-treated rats $(2 / 7$ died). None of the lidocaine-treated rats $(n=5)$ or CCPA plus lidocaine-treated rats $(n=6)$ died. Only data from surviving rats were further analyzed.

Ischemia-induced ventricular arrhythmias are shown in Figure 1. During ischemia, the saline control group experienced $130 \pm 64$ seconds of ventricular arrhythmia (VT, 89 \pm 41 seconds; VF, $41 \pm 26$ seconds), whereas CCPAtreated animals tolerated $56 \pm 18$ seconds of VT and no VF.
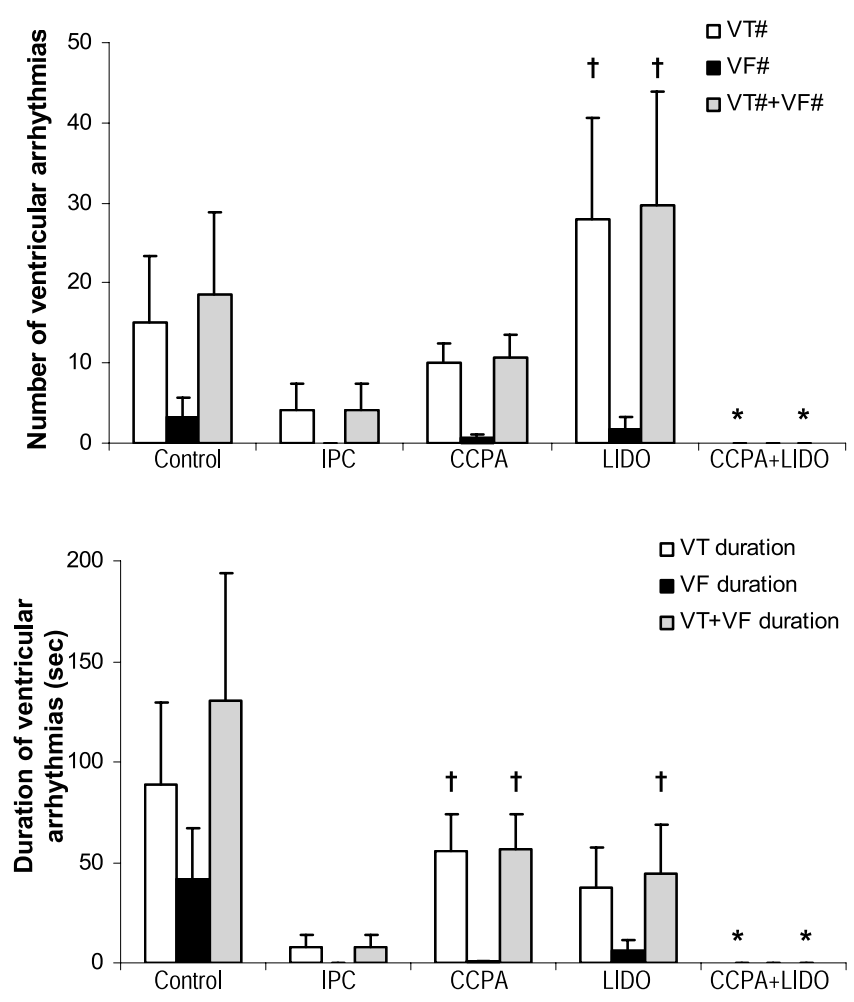

Figure 1. The episodes and duration of VT and VF and VT plus VF during ischemia for surviving rats in all treatment groups. These values represent the overall sum of episodes and durations (in seconds) that occurred throughout the 30 -minute ischemic period. Surviving rats: control, $n=6$; IPC, $n=5$; $A 1$ agonist only (CCPA), $n=5$; lidocaine only (LIDO), $n=5$; $A 1$ agonist plus lidocaine (CCPA+ LIDO), $\mathrm{n}=6$. Data are presented as means \pm SEM. ${ }^{*} P<$ .05 versus control animals. $+P<.05$ versus IPC-treated animals.

Forty percent of the IPC-treated rats experienced $4 \pm 3$ episodes of VT for $8 \pm 6$ seconds. Within the lidocaine group, $100 \%$ experienced VT $(28 \pm 13$ episodes, $38 \pm 20$ seconds), and $40 \%$ experienced VF ( $2 \pm 2$ episodes, $7 \pm 5$ seconds). Treatment with CCPA plus lidocaine completely abolished VT and VF (Figure 1). Immediately after ischemia, $80 \%$ of saline control rats, $60 \%$ of IPC-treated rats, $100 \%$ of CCPA-treated, and $20 \%$ of lidocaine-treated rats experienced reperfusion tachycardias. No ventricular arrhythmias during reperfusion were experienced in rats pretreated with CCPA plus lidocaine.

The mean AAR/LV, AN/LV, and AN/AAR are shown in Figure 2. The areas at risk expressed as a percentage of the left ventricle were not significantly different among the 5 groups and on average comprised $60 \% \pm 3 \%$. The areas of necrosis in saline control rats, IPC-treated rats, CCPAtreated rats, lidocaine-treated rates, and CCPA plus lidocainetreated rats were $31 \% \pm 2 \%, 7 \% \pm 2 \%, 24 \% \pm 3 \%, 35 \%$ $\pm 4 \%$, and $8 \% \pm 3 \%$, respectively. The mean infarct size 

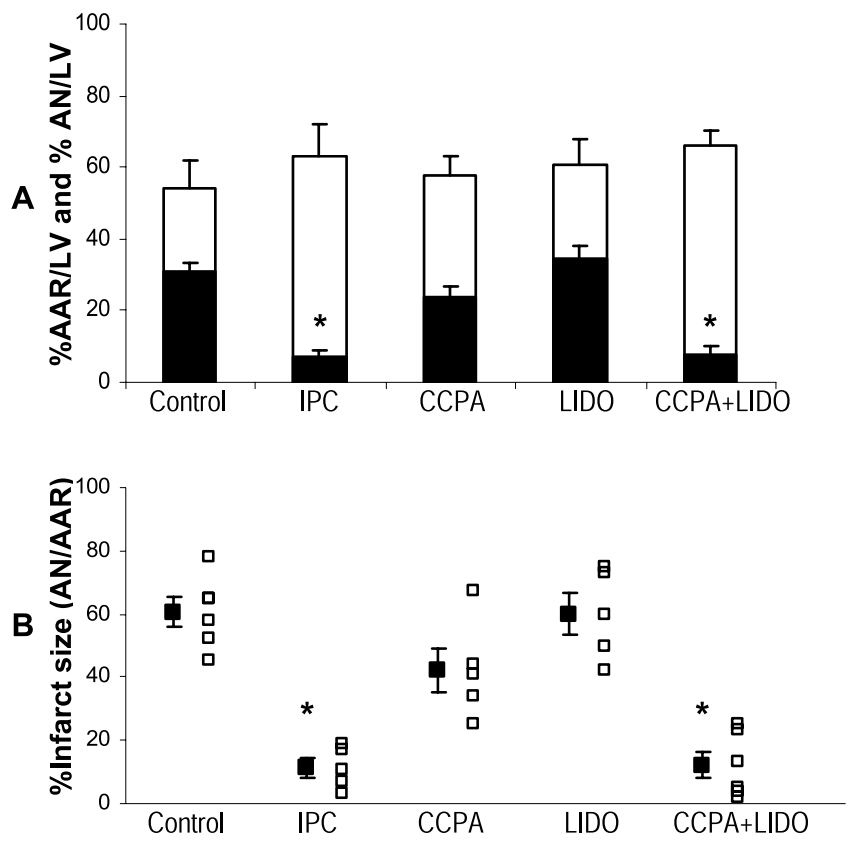

Figure 2. Effects of IPC, CCPA, lidocaine (LIDO), and CCPA plus lidocaine on left ventricular necrosis and infarct size. A, Areas at risk (AAR/LV, clear bars) were not significantly different between groups. Areas of necrosis in the left ventricle $(A N / L V$, filled bars) were significantly smaller with IPC and CCPA plus lidocaine treatment. $B$, Infarct sizes ( $A N / A A R)$ in groups receiving IPC and CCPA plus lidocaine treatment were significantly smaller compared with those of all other treatment groups. Surviving rats: control, $n=6$; IPC, $n=5$; A1 agonist only (CCPA), $n=5$; lidocaine only (LIDO), $\mathrm{n}=5$; $\mathrm{A1}$ agonist plus lidocaine $(C C P A+L I D O), n=6$. Data are means \pm SEM. ${ }^{*} P<.05$ versus control animals.

was $61 \pm 5 \%$ for saline controls rats, $11 \% \pm 3 \%$ for IPC-treated rats, $42 \% \pm 7 \%$ for CCPA-treated rates, $60 \% \pm$ $6 \%$ for lidocaine-treated rates, and $12 \% \pm 4 \%$ for CCPA plus lidocaine-treated rats (Figure 2). IPC and pharmacologic pretreating with CCPA plus lidocaine were both significantly reduced compared with that seen in control animals $(P<.05)$ but were not significantly different from one another.

The hemodynamic changes during pretreatment, ischemia, and reperfusion are shown in Table 1. For each treatment, MAP was lower during ischemia and significantly lower at 120 minutes of reperfusion compared with baseline values. MAPs in IPC-treated, CCPA-treated, lidocaine-treated, and CCPA plus lidocaine-treated rats were also lower before occlusion compared with their respective baseline values. Rats pretreated with CCPA plus lidocaine also experienced significant reductions in heart rate, MAP, and systolic pressure compared with values observed in saline control rats and IPC-treated rats. Al- though hemodynamic measurements were lower than baseline values after 2 hours of reperfusion in all groups, no group was significantly different from another, with the exception of those receiving only lidocaine. The Pearson correlation coefficient was analyzed for time points before the reperfusion period to assess whether changes in RPP influenced the final infarct size measurements. No significant correlations were found for any of the treatment groups tested, including control rats and IPC-treated rats.

\section{Discussion}

A goal for more than 2 decades has been to develop a pharmacologic mimetic of IPC to protect the heart during acute regional ischemia. Nearly every IPC study has shown a profound reduction in infarct size, and most have reported a large reduction in arrhythmias. However, some studies, including the original study of Murry and colleagues, 1,30 have reported a proarrhythmic effect of IPC, which might predispose the heart to stunning. ${ }^{31,32}$ Our report shows that coadministration of the A1 receptor agonist CCPA and the $\mathrm{Na}^{+}$fast-channel modulator lidocaine 5 minutes before and during 30 minutes of regional ischemia led to no deaths, no life-threatening arrhythmias, and a profound reduction in myocardial infarct size. Remarkably, the reduction of infarct size from $61 \%$ to $12 \%$ was equivalent to that achieved with IPC (Figure 2). Moreover, the combination of CCPA and lidocaine surpassed IPC protection by virtually abolishing ventricular arrhythmias and resulting in no deaths (Figure 1).

Previously, we have shown that constant infusion of AL had similar effects to CCPA and lidocaine but differed in the degree of infarct size reduction compared with that seen in saline control animals. ${ }^{25}$ Substituting the A1 agonist CCPA for adenosine reduced infarct size from $38 \%$ (AL) to $12 \%$. Thus we have shown that both ischemia therapies confer significant advantages to collectively protect against mortality, severe arrhythmias, and cell death compared with that seen in saline control rats, adenosine-treated rats, lidocaine-treated rats, or CCPA-treated rats (Figures 1 and 2). ${ }^{25}$ With the exception of lidocaine treatment, $29 \%$ of CCPA-treated rats and $50 \%$ of adenosine-treated rats died during ischemia (Figures 1 and 2). ${ }^{25}$ It was only the combination of CCPA plus lidocaine or AL solution that conferred an overall survival benefit, virtual abolition of severe arrhythmias, and reduction in infarct size. As mentioned in our earlier study with AL solution, ${ }^{25}$ the current protocol is very different from that of Homeister and associates, ${ }^{33}$ who administered an intravenous bolus of lidocaine in openchest dogs 1 minute before a 90-minute occlusion of the left circumflex coronary artery and again 1 minute before reperfusion, immediately followed by adenosine infusion at reperfusion and continued for 1 hour. In our studies CCPA plus lidocaine or AL was administered simultaneously 5 
TABLE 1. Hemodynamic parameters before and during ischemia and reperfusion and immediately after pretreatment before ischemia (before occlusion)

\begin{tabular}{|c|c|c|c|c|c|}
\hline Treatment & Parameter & Baseline & Before occlusion & $30 \mathrm{~min}$ of ischemia & $\begin{array}{l}120 \text { min of } \\
\text { reperfusion }\end{array}$ \\
\hline \multirow[t]{3}{*}{ Saline control } & HR (beats/min) & $450 \pm 17$ & $453 \pm 19$ & $416 \pm 33$ & $388 \pm 24$ \\
\hline & MAP (mm Hg) & $115 \pm 5$ & $118 \pm 7$ & $88 \pm 13$ & $75 \pm 8$ \\
\hline & Systolic blood pressure (mm Hg) & $143 \pm 6$ & $144 \pm 7$ & $106 \pm 6$ & $103 \pm 10$ \\
\hline \multirow[t]{3}{*}{ IPC } & HR (beats/min) & $438 \pm 9$ & $416 \pm 16$ & $414 \pm 7$ & $379 \pm 8$ \\
\hline & $\mathrm{MAP}(\mathrm{mm} \mathrm{Hg})$ & $130 \pm 8$ & $90 \pm 19$ & $92 \pm 14$ & $69 \pm 6$ \\
\hline & Systolic blood pressure (mm Hg) & $163 \pm 12$ & $116 \pm 22$ & $116 \pm 14$ & $98 \pm 6$ \\
\hline \multirow[t]{3}{*}{ ССРА } & HR (beats/min) & $421 \pm 15$ & $336 \pm 29^{*}$ & $382 \pm 4$ & $367 \pm 12$ \\
\hline & $\operatorname{MAP}(\mathrm{mm} \mathrm{Hg})$ & $114 \pm 6$ & $89 \pm 10$ & $91 \pm 10$ & $71 \pm 2$ \\
\hline & Systolic blood pressure (mm Hg) & $146 \pm 6$ & $113 \pm 11$ & $113 \pm 12$ & $94 \pm 4$ \\
\hline \multirow[t]{3}{*}{ LIDO } & HR (beats/min) & $440 \pm 19$ & $382 \pm 17$ & $305 \pm 27^{*} \dagger$ & $393 \pm 4$ \\
\hline & $\mathrm{MAP}(\mathrm{mm} \mathrm{Hg})$ & $120 \pm 10$ & $87 \pm 12$ & $57 \pm 5$ & $71 \pm 7$ \\
\hline & Systolic blood pressure (mm Hg) & $150 \pm 10$ & $115 \pm 12$ & $83 \pm 7$ & $99 \pm 93$ \\
\hline \multirow[t]{3}{*}{ CCPA + LIDO } & HR (beats/min) & $436 \pm 18$ & $270 \pm 14^{*} \dagger$ & $172 \pm 26^{*} \dagger$ & $347 \pm 17$ \\
\hline & $\mathrm{MAP}(\mathrm{mm} \mathrm{Hg})$ & $110 \pm 12$ & $49 \pm 4^{*}$ & $44 \pm 2^{*} \dagger$ & $72 \pm 5$ \\
\hline & Systolic blood pressure (mm Hg) & $131 \pm 9$ & $77 \pm 7^{*}$ & $59 \pm 3^{*} \dagger$ & $97 \pm 9$ \\
\hline
\end{tabular}

Data are presented as means \pm SEM. Surviving rats: saline control, $\mathrm{n}=6$; IPC, ischemic preconditioning $\mathrm{n}=5 ; C C P A, A 1$ agonist only, $\mathrm{n}=5 ;$ LIDO, lidocaine only, $\mathrm{n}=5$; CCPA+LIDO, $\mathrm{A} 1$ agonist plus lidocaine, $\mathrm{n}=6$. HR, Heart rate; $M A P$, mean arterial pressure. $* P<.05$ versus control. $\dagger P<.05$ versus IPC.

minutes before and during 30 minutes of regional ischemia and not separately and sequentially.

In an effort to understand how CCPA plus lidocaine (or AL solution) confers its cardioprotective effects, it is important to separate the direct effects of pretreatment on infarct size reduction and the indirect effects caused by hemodynamic changes. Hypotension, for example, might indirectly reduce infarct size by lowering left ventricular work. To tease apart this complex question, let us assume for a moment that the entire infarct size reduction from saline control animals (61\%) to AL-treated rats (38\%) reported by Canyon and Dobson ${ }^{25}$ was caused by hypotension. Because MAP was not significantly different between AL treatment (baseline, $125 \pm 11 \mathrm{~mm} \mathrm{Hg}$; before occlusion, $45 \pm 4 \mathrm{~mm} \mathrm{Hg} ; 25$ minutes of ischemia, $49 \pm 5 \mathrm{~mm} \mathrm{Hg}$; and 120 minutes of reperfusion, $86 \pm 6 \mathrm{~mm} \mathrm{Hg})^{25}$ and CCPA plus lidocaine treatment (baseline, $110 \pm 12 \mathrm{~mm} \mathrm{Hg}$; before occlusion, $49 \pm 4 \mathrm{~mm} \mathrm{Hg}$; 30 minutes of ischemia, $44 \pm 2 \mathrm{~mm} \mathrm{Hg}$; and 120 minutes of reperfusion, $72 \pm 5 \mathrm{~mm}$ $\mathrm{Hg}$; Table 1), the contribution of hypotension to infarct size reduction in CCPA plus lidocaine-treated rats could not exceed the decrease from $61 \%$ to $38 \%$ (ie for AL treatment). Thus the maximal contribution of hypotension to infarct size reduction from $61 \%$ to $12 \%$ in CCPA plus lidocainetreated rats would be $(61-38)(61-12) \times 100=47 \%$, with the remaining $53 \%$ reduction coming directly from the pharmacologic therapy itself. Of course, if our assumption that infarct size reduction in AL-treated rats (61\% to 38\%) was not caused by hypotension, then the direct effect of CCPA plus lidocaine infusion on infarct size reduction could conceivably approach $100 \%$. This suggestion is consistent with Casati and associates, ${ }^{34}$ who showed that the protective action of CCPA activation of A1 receptors in the in vivo rabbit model, which was independent of changes to hemodynamics, including MAP. De Jonge and colleagues ${ }^{9}$ have also shown in the isolated rat heart that the bradycardia effect of CCPA (Table 1) contributes little to infarct size reduction in the paced and nonpaced heart. More recently, Peart and Gross ${ }^{35}$ have ruled out the possibility of CCPA's bradycardic effect influencing infarct size reduction in paced rat hearts in vivo. Similarly, when RPP, an index of myocardial oxygen consumption, is plotted against infarct size, we found no significant correlations for CCPA or CCPA plus lidocaine-treated animals. However, for the lidocaine-only treatment, RPP at the end of the reperfusion period (120 minutes) was positively correlated with increasing infarct sizes; a higher RPP at 120 minutes led to a higher infarct size $(r=-0.90, P<.05)$ but not at any other times.

The underlying mechanisms for the cardioprotective effects of coadministering CCPA plus lidocaine (or AL solution) during ischemia are not currently known but are likely to occur at 3 integrated levels: electrophysiologic, mechanical, and metabolic. Electrophysiologically, the near-complete abolition of ventricular arrhythmias (Figure 1) might be linked to adenosine A1 receptor and lidocaine's ability to improve atrioventricular matching of conduction and pump performance. It is well known that adenosine, by activating A1 receptors, slows the sinoatrial nodal pacemaker rate, delays atrioventricular nodal impulse conduction, and reduces atrial contractility. ${ }^{36}$ Adenosine's net effect appears greatest at the atrioventricular 
node, where it is 30 times more effective in slowing conduction than sinoatrial pacemakers, which at therapeutic concentrations might reduce re-entry arrhythmias. ${ }^{37}$ Indeed, adenosine is chiefly used by clinicians to abate narrow complex supraventricular tachycardia but can also assist, to a lesser degree, in the management of VT of aberrant origin. ${ }^{37}$ As an antiarrhythmic, adenosine A1 agonist activation also inhibits the effect of catecholamines by reducing cyclic adenosine monophosphate and slowing $\mathrm{Ca}^{2+}$ influx ${ }^{36}$ and has been implicated in opening myocardial ATP-sensitive potassium channels, ${ }^{12,20,38}$ which might further stabilize the resting membrane potential and conduction inhomogeneities during contraction and relaxation. One or more of these actions might also be enhanced by lidocaine's effects on sodium channels and therefore excitability, which is potentiated in ventricular tissue by ischemic conditions. ${ }^{39}$ Lidocaine at low concentrations reduces voltage-dependent $\mathrm{Na}^{+}$entry and effectively resets the membrane potential to a more polarized voltage (ie, limit the reduction in ischemicinduced maximum diastolic potential) ${ }^{40}$ and, like adenosine, can shorten the action potential and thereby reduce $\mathrm{Ca}^{2+}$ entry into the cell. ${ }^{36}$ The overall effect could be to downregulate myocardial metabolism during ischemia and thereby improve pump performance in the postischemic period. Thus reduction of atrial and ventricular myocyte excitability, possible shortening of the action potential, and slowing of repolarization might explain how the combination of adenosine A1 receptor activation and lidocaine abolishes ventricular arrhythmias in the rat model and leads to a better electrical and metabolic matching in both ischemic and nonischemic regions of the heart. To this end, we have recently shown that ${ }^{31} \mathrm{P}$ nuclear magnetic resonancederived myocardial ATP levels are extremely stable during acute regional ischemia compared with ATP in the hearts of saline control animals, indicating an improved steady-state matching between supply and demand (work in progress).

In conclusion, we demonstrate a multitiered benefit of coadministering CCPA plus lidocaine before and during regional ischemia in the rat in vivo. At higher concentrations, the combination could also be used as a nondepolarizing surgical cardioplegia, as we have recently reported for AL. ${ }^{14,15}$ In addition, novel adenosine agonists, allosteric modifiers, or both or different pharmacologic mixtures of A1, A2, and A3 agonists and perhaps selective antagonists could be used with $\mathrm{Na}^{+}$channel modulation to protect and preserve organs and tissues in a number of clinical and surgical settings, including organ transplantation.

\section{Limitations to the Study Protocol}

A possible limitation of using intravenous infusions of A1 agonist plus lidocaine (or adenosine plus lidocaine) in the clinical setting might be the combination's effect on hemodynamics before occlusion and during regional ischemia (ie, decreased heart rate, MAP, or systolic pressure). Where acute systemic hypotension is to be avoided, ${ }^{7,17}$ adenosine A1 receptor activation plus lidocaine (or AL) therapy could be administered through an intracoronary route and used for percutaneous coronary interventions, off- and on-pump heart surgery, and perioperative applications, or alternatively, intravenous infusions could be considered at lower concentrations of one or both of the drugs in combination. Further studies are required to determine the appropriate dose-response curves in larger animals before clinical testing.

We thank Professor Jakob Vinten-Johansen and Professor Emeritus Arnold Katz for their helpful discussions on parts of the manuscript.

\section{References}

1. Murry CE, Jennings RB, Reimer KA. Preconditioning with ischemia: a delay of lethal cell injury in ischemic myocardium. Circulation. 1986;74:1124-36.

2. Kuzuya T, Hoshida S, Yamashita N, Fuji H, Oe H, Hori M, et al. Delayed effects of sublethal ischemia on the acquisition of tolerance to ischemia. Circ Res. 1993;72:1293-9.

3. Przyklenk K, Kloner RA. Ischemic preconditioning: exploring the paradox. Prog Cardiovasc Dis. 1998;40:517-47.

4. Bolli R. The late phase of preconditioning. Circ Res. 2000;87:972-83.

5. Baxter GF. Role of adenosine in delayed preconditioning of myocardium. Cardiovasc Res. 2002;55:483-94.

6. Zhao Z-Q, Corvera JS, Halkos ME, Kerendi F, Wang N-P, Guyton RA, et al. Inhibition of myocardial injury by ischemic post-conditioning during reperfusion: comparison with ischemic preconditioning. Am J Physiol Heart Circ Physiol. 2003;285:H579-588.

7. Yellon DM, Downey JM. Spotlight on preconditioning. Cardiovasc Res. 2002;55:425-8.

8. Lasley RD, Zhou Z, Hegge JO, Bunger R, Mentzer RM Jr. Adenosine attenuates in vivo myocardial stunning with minimal effects on cardiac energetics. Basic Res Cardiol. 1998;93:303-12.

9. De Jonge R, De Jong JW, Keijzer E, Bradamante S. The role of adenosine in preconditioning. Pharmacol Ther. 2000;87:141-9.

10. Toyoda Y, Friehs I, Parker RA, Levitsky S, McCully JD. Differential role of sarcolemmal and mitochondrial K(ATP) channels in adenosineenhanced ischemic preconditioning. Am J Physiol Heart Circ Physiol. 2000;279:H2694-703.

11. Avkiran M. Protection of the ischemic myocardium by $\mathrm{Na}+/ \mathrm{H}+$ exchange inhibitors: potential mechanisms of action. Basic Res Cardiol. 2001;96:306-11.

12. Gross GJ, Peart JN. KATP channels and myocardial preconditioning. Am J Physiol Heart Circ Physiol. 2003;285:H921-30.

13. Vinten-Johansen J. Involvement of neutrophils in the pathogenesis of lethal myocardial reperfusion injury. Cardiovasc Res. 2004;61:481-97.

14. Dobson GP, Jones MW. Adenosine and Lignocaine: a new concept in non-depolarising surgical arrest, protection and preservation. $J$ Thorac Cardiovasc Surg. 2004;127:794-805.

15. Dobson GP. Organ arrest, protection and preservation: natural hibernation to cardiac surgery: an invited review. Comp Biochem Physiol, Part B. 2004;139:469-85.

16. Vinten-Johansen J, Thourani VH, Ronson RS, Jordan JE, Zhao ZQ, Nakamura M, et al. Broad-spectrum cardioprotection with adenosine. Ann Thorac Surg. 1999;68:1942-8.

17. Yellon DM, Dana A. The preconditioning phenomenon: a tool for the scientist or clinical reality. Circ Res. 2000;87:543-50.

18. Cohen MV, Basines CP, Downey JM. Ischemic Preconditioning: from adenosine to KATP channel. Ann Rev Physiol. 2000;62:79-109.

19. Post H, Heusch G. Ischemic preconditioning: experimental facts and clinical perspective. Minerva Cardioangiol. 2002;50:569-605. 
20. Gross GJ, Fryer RM. Sarcolemmal versus mitochondrial ATP-sensitive K+ channels and myocardial protection. Circ Res. 1999;84:973-9.

21. O'Rourke B. Mitochondrial KATP channels in preconditioning. Circ Res. 2000;87:845-55.

22. Mubagwa K. Does adenosine protect the heart by acting on the sarcoplasmic reticulum. Cardiovasc Res. 2002;53:286-9.

23. Carmeliet E. Cardiac ionic channels and acute ischemia: from channels to arrhythmias. Physiol Rev. 1999;79:917-1017.

24. Balser JR. The cardiac sodium channel: gating function and molecular pharmacology. J Mol Cell Cardiol. 2001;33:599-613.

25. Canyon SJ, Dobson GP. Protection against ventricular arrhythmias and cardiac death using adenosine and lidocaine during regional ischemia in the in vivo rat. Am J Physiol Heart Circ Physiol. 2004;287:H128695 .

26. Lasley RD, Rhee JW, Van Wylen DG, Mentzer RM. Adenosine A1 receptor mediated protection of the globally ischemic rat heart. $J \mathrm{Mol}$ Cell Cardiol. 1990;22:39-47.

27. Gao ZG, Jacobson KA. 2-Chloro-N(6)-cyclopentyladenosine, adenosine $\mathrm{A}(1)$ receptor agonist, antagonizes the adenosine $\mathrm{A}(3)$ receptor. Eur J Pharmacol. 2002;443:39-42.

28. Walker MJA, Curtis MJ, Hearse DJ, Campbell RWF, Janse MJ, Yellon DM, et al. The Lambeth Convention: guidelines for the study of arrhythmias in ischaemia, infarction, and reperfusion. Cardiovasc Res. 1988;22:447-55.

29. Fishbein MC, Meerbaum S, Rit J, Lando U, Kanmatsuse K, Mercier JC, et al. Early phase acute myocardial infarct size quantification: validation of the triphenyltetrazolium chloride tissue enzyme staining technique. Am Heart J. 1981;101:593-600.

30. Ovize M, Aupetit JF, Riofol G, Loufoua J, Andre-Fouet X, Minaire Y, et al. Ischemic preconditioning reduces infarct size but accelerates time to ventricular fibrillation in ischemic pig heart. Am J Physiol Heart Circ Physiol. 1995;269:H72-9.

31. Mentzer RM. Ischemic preconditioning: how close are we to therapeutic implementation? Ann Thorac Surg. 2000;70:356-7.

32. Taggart P, Yellon DM. Preconditioning and arrhythmias. Circulation. 2002;106:2999-3001.

33. Homeister JW, Hoff PT, Fletcher DD, Lucchesi BR. Combined adenosine and lidocaine administration limits myocardial reperfusion injury. Circulation. 1990;82:595-608.

34. Casati C, Forlani A, Lozza G, Monopoli A. Hemodynamic changes do not mediate the cardioprotection induced by the A1, adenosine receptor agonist CCPA in the rabbit. Pharmacol Res. 1997;35:51-5.

35. Peart JN, Gross GJ. Adenosine and opioid receptor-mediated cardioprotection in the rat: evidence for cross talk between receptors. Am J Physiol Heart Circ Physiol. 2003;285:H81-9.

36. Lerman BB, Belardinelli L. Cardiac electrophysiology of adenosine. Basic and clinical concepts. Circulation. 1991;83:1499-509.

37. Pelleg A, Kutalek SP. Adenosine in the mammalian heart: nothing to get excited about. Trends Pharmacol Sci. 1997;18:236-8.

38. Kirsch GE, Codina J, Birnbaumer L, Brown AM. Coupling of ATP sensitive $\mathrm{K}+$ channels to $\mathrm{A} 1$ receptors by $\mathrm{G}$ proteins in rat ventricular myocytes. Am J Physiol Heart Circ Physiol. 1990;259:H820-6.

39. Li G-R, Ferrier GR. Effects of lidocaine on reperfusion arrhythmias and electrophysiological properties in an isolated ventricular muscle model of ischemia and reperfusion. J Pharmacol Exp Ther. 1991;257: 997-1004.

40. Barrett TD, Hayes ES, Yong SL, Zolotoy AB, Abraham S, Walker MJA. Ischaemia selectivity confers efficacy for suppression of ischaemia-induced arrhythmias in rats. Eur J Pharmacol. 2000;398:36574. 